\title{
Role of tip size, orientation, and structural relaxations in first-principles studies of magnetic exchange force microscopy and spin-polarized scanning tunneling microscopy
}

\author{
C. Lazo, ${ }^{1, *}$ V. Caciuc, ${ }^{2}$ H. Hölscher, ${ }^{3}$ and S. Heinze ${ }^{1}$ \\ ${ }^{1}$ Institute of Applied Physics and Microstructure Research Center, University of Hamburg, Jungiusstrasse 11, \\ 20355 Hamburg, Germany \\ ${ }^{2}$ Institut für Festkörperforschung (IFF), Foschungszentrum Jülich, 52425 Jülich, Germany \\ ${ }^{3}$ Institute for Microstructure Technology, Forschungszentrum Karlsruhe, P.O. Box 36 70, 76021 Karlsruhe, Germany \\ (Received 29 September 2008; revised manuscript received 10 November 2008; published 11 December 2008)
}

\begin{abstract}
Using first-principles calculations based on density-functional theory, we investigated the exchange interaction between a magnetic tip and a magnetic sample which is detected in magnetic exchange force microscopy (MExFM) and also occurs in spin-polarized scanning tunneling microscopy (SP-STM) experiments. As a model tip-sample system, we chose Fe tips and one monolayer Fe on W(001) which exhibits a checkerboard antiferromagnetic structure and has been previously studied with both SP-STM and MExFM. We calculated the exchange forces and energies as a function of tip-sample distance using different tip models ranging from single $\mathrm{Fe}$ atoms to Fe pyramids consisting of up to fourteen atoms. We find that modeling the tip by a single Fe atom leads to qualitatively different tip-sample interactions than using clusters consisting of several atoms. Increasing the cluster size changes the calculated forces, quantitatively enhancing the detectable exchange forces. Rotating the tip with respect to the surface unit cell has only a small influence on the tip-sample forces. Interestingly, the exchange forces on the tip atoms in the nearest and next-nearest layers from the apex atom are non-negligible and can be opposite to that on the apex atom for a small tip. In addition, the apex atom interacts not only with the surface atoms underneath but also with nearest neighbors in the surface. We find that structural relaxations of tip and sample due to their interaction depend sensitively on the magnetic alignment of the two systems. As a result the onset of significant exchange forces is shifted toward larger tip-sample separations which facilitates their measurement in MExFM. At small tip-sample separations, structural relaxations of tip apex and surface atoms can either enhance or reduce the magnetic contrast measured in SP-STM.
\end{abstract}

DOI: 10.1103/PhysRevB.78.214416

PACS number(s): 75.30.Et, 75.70.Ak, 68.37.Ps, 75.70.Rf

\section{INTRODUCTION}

Recent advances in magnetic microscopy techniques ${ }^{1,2}$ have allowed fascinating new insights into magnetic properties of nanostructures at surfaces. Such experimental measurements challenge and drive the theoretical understanding of magnetism in reduced dimensions and are crucial in developing new magnetic materials. Among these experimental methods, the spin-polarized scanning tunneling microscope (SP-STM) plays a central role as it has opened the possibility of imaging magnetic structures down to the atomic scale. ${ }^{3-6}$ More recently, the feasibility to measure even exchange forces between a magnetic tip and a magnetic sample directly has been demonstrated using an atomic force microscope (AFM) equipped with a magnetically coated tip. ${ }^{7}$ This technique, denoted as magnetic exchange force microscopy (MExFM) (Ref. 8) opens new vistas in atomic-scale magnetic imaging 9 as it is applicable to all magnetic surfaces, i.e., conducting as well as insulating systems, ${ }^{7}$ e.g., magnetic molecules. However, as in SP-STM the interpretation of measurements by MExFM is not straightforward and the development of theoretical models and tools to understand them is essential.

Scanning probe-microscopy techniques are capable of operating at the atomic scale and, if they are spin sensitive, down to the single spin level. Thus, quantum-mechanical effects on the microscopic level are crucial and need to be properly taken into account to achieve a theoretical understanding of these techniques. In the past, first-principles cal- culations based on density-functional theory (DFT) have demonstrated their great potential in this respect. In fact, they have become indispensable and versatile tools in studying real nanostructures in order to gain a qualitative and often even quantitative understanding. However, one is frequently limited by the size of the system which can be considered and the level of approximation which is used.

In the case of scanning tunneling microscopy (STM), the Tersoff-Hamann mode ${ }^{10}$ and its generalization to spinpolarized tunneling ${ }^{11}$ is most often used to calculate and interpret experimental images. However, the interactions with the tip are neglected in this model. Therefore, it is only necessary to calculate the electronic and magnetic structure of the isolated sample, in particular, the local density of states at a few Angströms above the sample surface. Naturally, the approximation of the Tersoff-Hamann model breaks down at small tip-sample distances. Effects of tip-sample interaction on the tunneling current have been investigated theoretically in the past concerning conventional STM (Refs. 12-14) but, to our knowledge, not with respect to SP-STM.

On the other hand, to model atomic force microscopy experiments, it is essential to calculate the forces between the sample and the tip. For this purpose one has to include, besides the sample, some kind of tip model in the calculations. This fact makes the simulation of AFM experiments much more challenging, in particular, if one allows for structural relaxations of tip and sample which can often be crucial. Such realistic theoretical modeling of the interaction between tip and sample, and even of the entire experimental proce- 
dure, has become an integral and essential part of many AFM experiments. ${ }^{15-25}$ Nevertheless, in the case of MExFM, there have been only few studies in the past. ${ }^{26-31}$

The first theoretical study of MExFM was based on a semiempirical tight-binding calculation. ${ }^{26}$ It was shown that the exchange forces between an iron tip and a chromium or a nickel surface are well below $1 \mathrm{nN}$ but they should be detectable with an atomic force microscope. The authors found that the exact morphology of the tip does not play an important role on the results. In this work, however, relaxation of the apex atom and the sample were neglected, and only the $d$ electrons of the system were considered. Subsequently, Nakamura et al. ${ }^{27,28}$ employed a more sophisticated approach based on DFT to calculate the magnetic exchange force between two $\mathrm{Fe}(001)$ surfaces. Forces of a few $\mathrm{nN}$ were obtained at a distance of $3 \AA$. Additionally, the forces exhibited an oscillatory RKKY-interaction-like behavior as a function of separation. Even above $4 \AA$, the measured forces were still within the experimental resolution limit of AFM. Later, Foster and Shluger ${ }^{29}$ used a periodic unrestricted HartreeFock method to calculate the interaction of a spin-polarized $\mathrm{H}$ or $\mathrm{Na}$ atom with the antiferromagnetic $\mathrm{NiO}(001)$ surface. They found that the difference in force over $\mathrm{Ni}$ atoms with opposite spins should be detectable with the AFM for a tipsample distance smaller than $4 \AA$ or for imaging close to the repulsive regime. However, at such short distances, the chemical forces can become strong and it was speculated that instabilities may become apparent.

A more recent first-principles study ${ }^{30,31}$ of the exchange force between a single iron atom, representing the tip, and the (001) surface of the antiferromagnetic insulator $\mathrm{NiO}$ has been carried out within the framework of DFT. The calculated MExFM images show a magnetic contrast on the atomic scale when the single-atom Fe tip approaches the surface within $1 \AA$ above the contact point. Therefore, this work predicted the possibility of using AFM for magnetic imaging with atomic resolution. However, this study did not address the role of structural relaxations and the adequacy of the single-atom tip model was not investigated.

Nonetheless, these early theoretical predictions and the outlook to directly measure magnetic exchange forces encouraged many experimental attempts to demonstrate MExFM, focusing especially on the (001) surface of the antiferromagnetic insulator $\mathrm{NiO} .{ }^{32-36}$ However, it took several years before the first successful experiment demonstrating the predicted effect was reported. 7,37

Here, we apply density-functional theory using the fullpotential linearized augmented plane-wave (FLAPW) method to study the interaction of a magnetic tip and a magnetic sample as it occurs in SP-STM or MExFM. We consider one monolayer (ML) Fe on $\mathrm{W}(001)$ as a model sample system which exhibits a $c(2 \times 2)$ antiferromagnetic structure and has been experimentally resolved by both SP-STM (Ref. 6) and MExFM (Ref. 38). The iron tip is modeled by a single Fe atom as well as by Fe clusters of different sizes, and structural relaxations of both tip and sample due to their mutual interaction are also included. Our results show that the relaxations depend sensitively on the magnetic configuration between tip and sample, i.e., whether the tip magnetization is parallel or antiparallel to the Fe atom below. We calculate the exchange forces and demonstrate that their measurement in MExFM for this tip/sample system is facilitated due to relaxations as their onset is shifted to larger tip-sample separations. By simulating MExFM images, one can explain the contrasts observed in recent experiments and show that they are due to a competition between chemical and magnetic forces. ${ }^{38}$

Concerning SP-STM, we estimate the effect of tip-sample relaxations on the experimental corrugation amplitude, i.e., the maximum vertical tip height change while scanning the surface in the constant-current mode. We find that at a tipsample separation of $4 \AA$ the corrugation amplitude due to relaxations is of similar magnitude as the contribution from the spin-polarized tunneling current. This corrugation amplitude due to the exchange forces can either enhance or reduce the total magnetic signal as the sign of the spin-polarized tunneling current depends on the electronic structure of tip and sample at the Fermi energy while the forces depend on the total, i.e., energy integrated, magnetization densities.

This paper is structured as follows. In Sec. II, we provide details on the computational method and setup of the calculations, e.g., the different geometries of the considered $\mathrm{Fe}$ tips are presented. In Sec. III we review the results. First, we discuss the obtained forces on a pyramid of five $\mathrm{Fe}$ atoms without structural relaxations due to tip-sample interaction. We then consider the effect of rotating the tip and analyze the contributions of total force originating from the different tip atoms. From these force curves we expect considerable structural relaxations of tip and sample due to their mutual interaction. In Sec. III B, the relaxations are shown to depend on the local magnetic configuration between tip and sample magnetizations, and we find that the onset of significant exchange forces is shifted to larger tip-sample distances. In Sec. III C, we analyze the tip-sample interaction in terms of magnetic moments and charge-density difference plots which clearly indicate that there is an interaction of the tip apex atom with nearest and next-nearest Fe surface atoms. In Sec. III D, we compare the exchange forces obtained with a fiveatom $\mathrm{Fe}$ tip to calculations using a single $\mathrm{Fe}$ atom or a fourteen-atom Fe tip. Qualitatively, the exchange interaction is similar for the two cluster tips while the single-atom Fe tip seems an inappropriate tip model. Finally, we estimate in Sec. III E the influence of tip and sample relaxations on the corrugation amplitude obtained in the constant-current mode of SP-STM.

\section{COMPUTATIONAL METHOD}

In order to gain insight into the magnetic interactions which occur in an SP-STM or MExFM experiment between an Fe tip and a monolayer of iron atoms on W(001), we have performed first-principles calculations based on densityfunctional theory within the generalized gradient approximation (GGA) (Ref. 39) to the exchange-correlation potential. We apply the full-potential linearized augmented plane-wave method as implemented in the WIEN2K (Ref. 40) code.

We used ferromagnetic Fe pyramids in bcc-(001) orientation of different size ranging from one to fourteen atoms to model the tip as shown in Fig. 1. The five-atom tip has been 
14 atoms
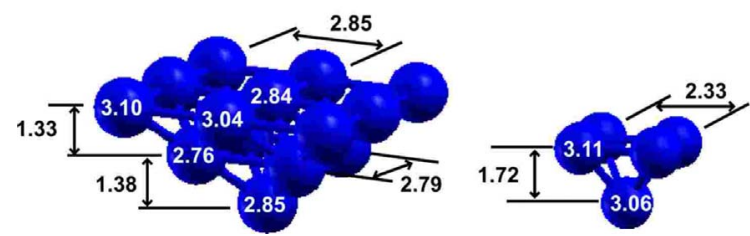

FIG. 1. (Color online) Different cluster geometries used to model the iron tip. The magnetic moments of the $\mathrm{Fe}$ atoms integrated over the muffin-tin spheres of radius $R_{\mathrm{MT}}=2.15$ a.u. are indicated in white in units of the Bohr magneton, $\mu_{B}$. Additionally, the interlayer distances are indicated in black in units of Ångström. The values correspond to relaxed geometries of the isolated tip, i.e., without any tip-sample interactions.

fully relaxed, i.e., also the in-plane separation between the base atoms, while for the fourteen-atom tip only the apex atom and the four atoms of the adjacent layer have been relaxed. The in-plane interatomic distance between the base atoms of the fourteen-atom tip has been kept fixed at the calculated GGA lattice constant of $\mathrm{Fe}(2.85 \AA)$. As can be seen in Fig. 1, the magnetic moments of the Fe apex atom is reduced significantly with increasing tip size from a single atom, $3.58 \mu_{B}$, to a fourteen-atom tip, $2.85 \mu_{B}$, which is very similar to the moment of $2.79 \mu_{B}$ we obtained for a single $\mathrm{Fe}$ atom adsorbed on the (001) surface of Fe.

The coupled system of tip and sample was calculated in a supercell geometry, as shown in Fig. 2 for the example of the five-atom Fe tip. The monolayer of Fe on W(001) was modeled by a symmetric slab with five layers of $\mathrm{W}$ atoms and one layer of $\mathrm{Fe}$ atoms on each side. We used the GGA lattice constant of W (3.181 $\AA$ ) which is only $0.5 \%$ larger than the experimental value (3.165 $\AA$ ). The muffin-tin radii of Fe and $\mathrm{W}$ are 2.15 and 2.50 a.u., respectively. The energy cutoff for the plane-wave representation in the interstitial region is $E_{\max }^{\mathrm{wf}}=11 \mathrm{Ry}$ and $\mathrm{a}(3 \times 3 \times 1)$ Monkhorst-Pack grid was used for the Brillouin-zone integration. Tip and surface were initially relaxed independently before considering the coupled system, i.e., the tip-sample interaction.

In two dimensions (2D) our supercell corresponds to a $c(4 \times 4)$ unit cell with respect to the $\mathrm{Fe} / \mathrm{W}(001)$ surface, as shown in Fig. 2(a) for the example of the five-atom tip. This choice guarantees that the tip interaction with its lateral image is negligible. For example, the lateral distances between adjacent five-atom tips are $9.0 \AA$ for the apex atom and $6.7 \AA$ for the base atoms of the tip. Our supercell is periodic also in $z$ direction. Choosing a very large vacuum separation of $21 \AA$ between adjacent surfaces, however, allows the tip to approach the surface without interacting with its periodic image.

The separation $z$ in the unrelaxed coupled system is defined as the distance between the centers of the tip apex atom and surface atom underneath. For the relaxed system the separation $z$ is defined as the distance between the center of the tip apex and surfaces atoms for the case of zero relaxation. As it turned out, the relaxation of these atoms are relatively small. Therefore, treating $z$ as a nominal distance (as if there were no relaxations at all) does not modify the force-distance curves much. In fact, it is reminiscent of the (a)

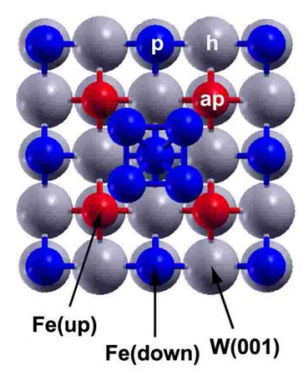

(b)

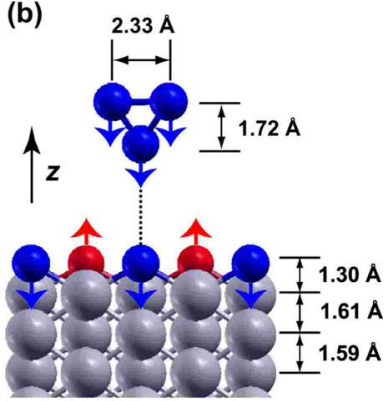

(c)

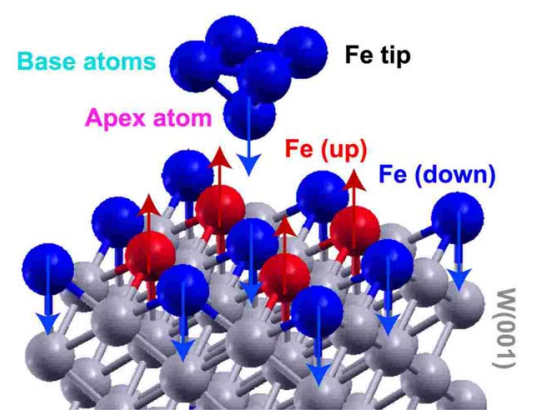

FIG. 2. (Color online) (a) Top view of the $c(4 \times 4)$ unit cell used to calculate the forces between the five-atom $\mathrm{Fe}$ pyramid and the $\mathrm{Fe}$ monolayer on W(001). Sites with parallel (p site) and antiparallel (ap site) alignments between tip and surface Fe magnetic moments (indicated by arrows) are marked as well as the hollow site (h-site). (b) Distances given in the side view are obtained after relaxing the tip and sample independently. $z$ is defined as tip-sample distance along the approach trajectory (dotted line) before considering relaxations due to tip-sample interactions. (c) 3D view of the unit cell.

experimental situation where the exact measurement of the distance between the tip apex and surface atom is impossible, and one uses some reference distance.

Force curves are calculated on two high-symmetry points of the surface, which are magnetically different with respect to the magnetization direction of the iron tip pyramid: on top of an Fe atom with parallel magnetic moment, $F_{\mathrm{p}}(z)$ (p site), and on top of an $\mathrm{Fe}$ atom with antiparallel magnetic moment, $F_{\text {ap }}(z)$ (ap site).

In the case of the five-atom tip, we also investigated the effect of rotating the tip by $45^{\circ}$ with respect to the $z$ axis, and the influence of structural relaxations of tip and sample due to their mutual interaction. Upon approaching the tip to the surface along the $z$ direction indicated by a dotted line in Fig. 2(b), we allowed all $\mathrm{Fe}$ atoms of the monolayer, the first layer of $\mathrm{W}$ atoms, and the $\mathrm{Fe}$ apex atom to relax at every tip-sample distance $z$. The remaining $z$ components of the forces acting on the base atoms constitute the total force on the tip.

\section{RESULTS}

In the following we present the results of our firstprinciples simulations of magnetic exchange force microscopy on the Fe monolayer on W(001). In Secs. III A-III C, we focus on the five-atom $\mathrm{Fe}$ tip comparing calculations 

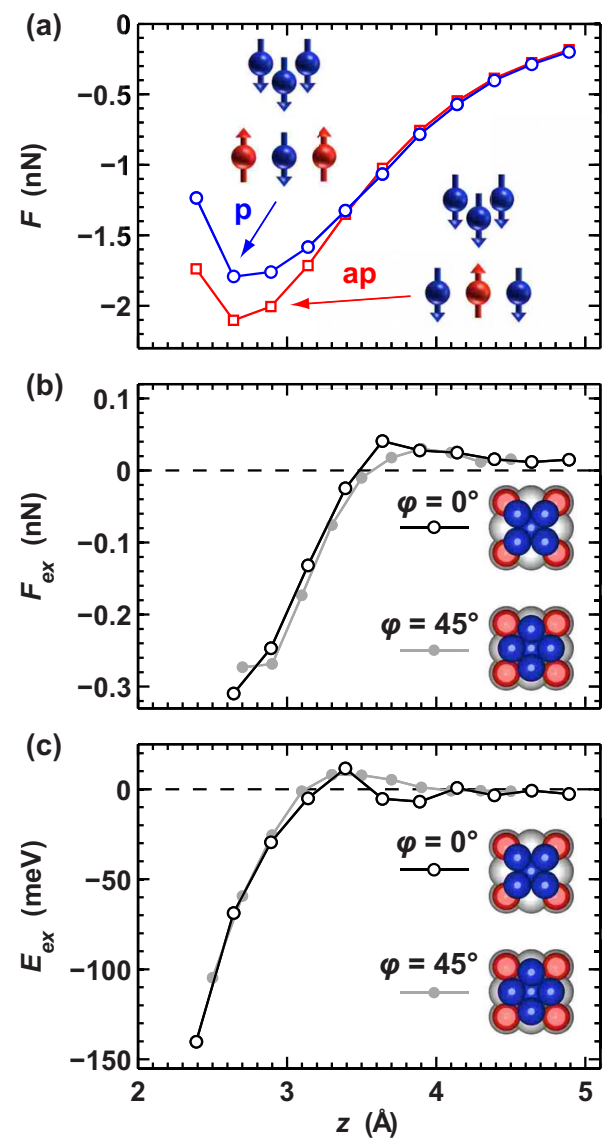

FIG. 3. (Color online) (a) Calculated force curves for the fiveatom Fe tip as it is approached to the Fe ML on W(001) on the ap site, $F_{\text {ap }}(z)$, and on the p site, $F_{\mathrm{p}}(z)$, of the surface (cf. Fig. 2) for the tip-sample system neglecting relaxations due to the interaction. (b) The resulting exchange force, $F_{\mathrm{ex}}(z)=F_{\mathrm{ap}}(z)-F_{\mathrm{p}}(z)$, and (c) the exchange energy $E_{\mathrm{ex}}(z)=E_{\mathrm{ap}}(z)-E_{\mathrm{p}}(z)$, as a function of the separation, $z$, between the tip apex atom and the approached Fe surface atom. The results for a tip rotated by $45^{\circ}$ are plotted by filled symbols.

without and with structural relaxations, and analyze the electronic structure changes due to the interaction. These results are compared in Sec. III D with calculations for a singleatom Fe tip and a fourteen-atom Fe tip. Finally, we discuss the implications of tip-sample interactions on spin-polarized STM measurements in Sec. III E.

\section{A. Unrelaxed tip and sample}

First, we performed separate structural relaxations of tip and sample [cf. Fig. 2(b)]. Then the tip was approached vertically to the surface of the sample on the p and ap sites [cf. Fig. 2(a)], keeping the internal geometry of the tip and sample fixed, i.e., neglecting structural relaxations due to the mutual interaction. The calculated total forces acting on the five-atom Fe tip are shown in Fig. 3(a). They display an attractive interaction for the ap and $\mathrm{p}$ alignments up to a maximum force of approximately -2.1 and $-1.8 \mathrm{nN}$, respectively, at about $2.7 \AA$. The difference between the force curves on the $\mathrm{p}$ and ap sites, clearly visible in Fig. 3(a), is the magnetic exchange force $(\mathrm{MExF}), F_{\text {ex }}(z)$, defined as

$$
F_{\text {ex }}(z)=F_{\text {ap }}(z)-F_{\mathrm{p}}(z),
$$

which is depicted in Fig. 3(b). Interestingly, the exchange force changes its sign upon approaching the surface and reaches significant values on the order of $0.2 \mathrm{nN}$ at about $3 \AA$. The negative sign of the exchange force indicates a more attractive interaction for an antiparallel alignment of the magnetization of the tip and the Fe surface atom which is being approached (ap site). The magnetic interaction between tip and sample can be inferred more directly from the magnetic exchange energy given by

$$
E_{\mathrm{ex}}(z)=E_{\mathrm{ap}}(z)-E_{\mathrm{p}}(z),
$$

and displayed in Fig. 3(c). At large tip-sample distances, the exchange energy is very small and positive while it becomes quite large and negative at small separations. Figures 3(b) and $3(\mathrm{c})$ include also the result of $F_{\mathrm{ex}}(z)$ and $E_{\mathrm{ex}}(z)$ for the cluster tip rotated by $45^{\circ}$ with respect to the horizontal (see Fig. 2). The results show that the magnetic exchange force and energy are hardly affected by rotating the cluster tip.

A negative sign of the exchange energy reveals that antiparallel alignment of tip and sample magnetizations, i.e., antiferromagnetic coupling, is energetically more favorable. This result may seem rather surprising at first glance as one would intuitively expect ferromagnetic coupling between the interacting $\mathrm{Fe}$ atoms of tip and sample. However, as we will show in Sec. III C the Fe apex atom interacts not only with the $\mathrm{Fe}$ surface atom beneath it but also with the four nearest Fe neighbors of this surface atom [cf. Fig. 2(c)]. Since the magnetic moments of the Fe atoms on the W(001) surface form an antiferromagnetic checkerboard structure, on the ap site the magnetization of the tip apex atom is aligned antiparallel to the moment of the Fe surface atom beneath it and parallel to the moments of the four nearest-neighbor Fe surface atoms, and vice versa on the p site [cf. Fig. 2]. Therefore, if we assume ferromagnetic coupling between individual $\mathrm{Fe}$ atoms, there is a competition of exchange interactions with the surface $\mathrm{Fe}$ atom and its nearest neighbors.

Further insight into the tip-sample interaction and the forces acting in the system can be obtained by decomposing the total force on the tip. The total force acting on the cluster tip, shown in Fig. 3(a), is the sum of the $z$ components of the forces acting on the tip apex atom and the four tip base atoms which are displayed in Figs. 4(a) and 4(b), respectively. The apex and base tip atoms are depicted in Fig. 2. The forces acting on the tip apex atom are qualitatively very similar to the total forces acting on the entire cluster. However, the splitting between the forces on the ap and $\mathrm{p}$ sites is dramatically enhanced. Consequently, these large force differences due to the magnetic interaction, result in site dependent relaxations of the tip apex atom-an effect we will study in the next section.

The forces summed over all four base atoms, shown in Fig. 4(b), look somewhat different than those acting on the apex atom. They can be understood if we take into account that we sum over four atoms and that the base atoms are $1.72 \AA$ farther from the surface atoms than the apex atom [cf. Fig. 1], and consequently do not come as close to the 

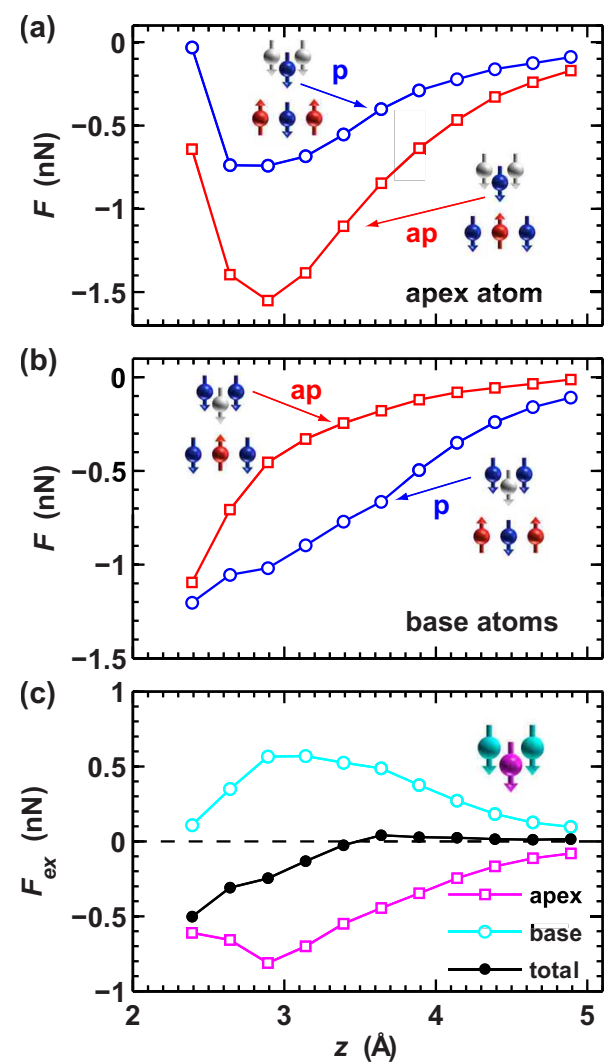

FIG. 4. (Color online) Decomposition of forces acting on the unrelaxed tip for the ap and p configurations. Forces acting on (a) the tip apex atom, on (b) the base of the cluster tip, and (c) the corresponding exchange forces.

surface. Therefore, the force curves of the base atoms should resemble only the part of the apex force curves at large distances, i.e., only the part of Fig. 4(a) up to about $z=4 \AA$, which is well fulfilled. Surprisingly, however, the forces on the base atoms are larger on the $\mathrm{p}$ site than on the ap site and therefore opposite to those on the apex atom.

The difference between the apex atom forces on the $p$ and ap sites can be interpreted as the individual exchange force acting on the apex tip atom, i.e.,

$$
F_{\mathrm{ex}}^{\mathrm{apex}}(z)=F_{\mathrm{ap}}^{\mathrm{apex}}(z)-F_{\mathrm{p}}^{\mathrm{apex}}(z) .
$$

A similar equation holds for the base atoms. As seen in Fig. 4(c), the MExF for the apex and base atoms have opposite sign and are considerably larger than their sum, i.e., total exchange force acting on the tip [cf. Fig. 3(c)]. The partial compensation of the exchange force on the tip apex and the four base atoms leads to a significant reduction in the total exchange force. In addition, the exchange force on the apex atom sets in already at much larger tip-sample distances and increasing the contribution from the apex atom would greatly enhance the measurable magnetic signal. This result reveals the influence of the interaction of the sample with the tip base atoms. A realistic model of the tip should therefore include not only a single tip apex atom but at least some tip base atoms. In Sec. III D, we will explore this aspect in more detail.
The shape of the exchange force curve for the Fe apex atom displayed in Fig. 4(c) also hints at competing exchange interactions of different signs, i.e., antiferromagnetic and ferromagnetic interactions. The force is negative at large tipsample separations, rises in magnitude with decreasing distance and reaches a local maximum of its absolute value at $z=2.9 \AA$ before the magnitude decreases again. A similar shape is visible for the base atoms but with opposite sign of the exchange force.

We can rationalize these force curves by assuming ferromagnetic exchange coupling between individual $\mathrm{Fe}$ atoms of tip and sample, and summing over the pairwise exchange interactions. At large tip-sample separations, the distance between the apex atom and the $\mathrm{Fe}$ surface atom beneath it is not much smaller than the distance of the apex atom to the four neighboring Fe surface atoms. Therefore, the ferromagnetic coupling of the Fe apex atom with the four neighboring surface $\mathrm{Fe}$ atoms can dominate over the interaction with the single $\mathrm{Fe}$ atom beneath the tip apex. An antiparallel alignment with respect to the $\mathrm{Fe}$ surface atom beneath the tip is then favorable. This situation results in a negative exchange force [cf. Eq. (1)], which increases with decreasing distance due to the larger wave-function overlap. At small tip-sample separations, however, the direct ferromagnetic coupling of the apex atom with the Fe surface atom beneath it becomes large and a parallel alignment is preferred. This leads to a positive contribution to the exchange force on the apex and the decrease in the exchange force at small separations. Of course, this simple discussion neglects that the exchange interaction between individual $\mathrm{Fe}$ atoms has a distance dependence of its own. In addition, the magnetic moments of the $\mathrm{Fe}$ atoms are not constant upon the approach of the tip, as we will see in Sec. III C.

\section{B. Influence of structural relaxations}

The calculations without structural relaxations presented in the last section for the five-atom tip showed that significant forces act on the tip apex atom depending on the magnetic configuration between tip and sample. From these results we conclude that relaxations of tip and sample due to the magnetic interactions can play an important role for the total detectable exchange force. Therefore, we carried out the same set of calculations as before but this time we performed a structural relaxation of the tip apex atom and the first two layers of the sample at every tip-sample separation. Since the tip apex atom is allowed to relax, the detectable force acting on the entire tip is given by the $z$ component of the force on the tip base atoms.

The obtained forces acting on the tip are shown in Fig. 5(a) for the calculation including relaxations due to tipsample interactions. They look qualitatively similar as the forces for the unrelaxed structure [cf. Fig. 3(a)]; however, quantitative differences arise in their respective exchange forces which can easily be observed in the splitting between the force curves on the $\mathrm{p}$ and ap sites. Upon including relaxations, the onset of large magnetic exchange forces shifts toward larger tip-sample distances as seen in Fig. 5(b). This effect facilitates their experimental detection as the atomic 

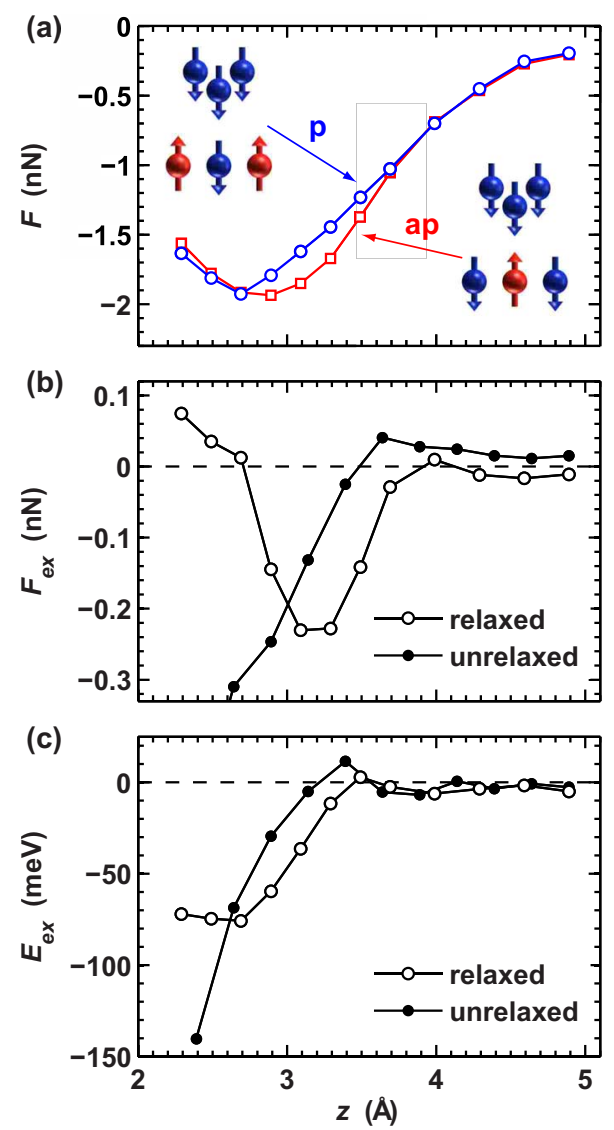

FIG. 5. (Color online) (a) Calculated force curves for the fiveatom Fe tip as it approaches the Fe ML on W(001) on the ap site, $F_{\text {ap }}(z)$, and on the p site, $F_{\mathrm{p}}(z)$, including relaxations of tip and sample due to their interaction. (b) Comparison of the magnetic exchange forces, $F_{\text {ex }}(z)=F_{\text {ap }}(z)-F_{\mathrm{p}}(z)$, and (c) magnetic exchange energies, $E_{\mathrm{ex}}(z)=E_{\mathrm{ap}}(z)-E_{\mathrm{p}}(z)$, between the calculations with and without relaxations as a function of the separation between the unrelaxed tip apex and probed Fe surface atom.

force microscope can be operated at larger distances from the point where a snap-to-contact can occur. In addition, $F_{\mathrm{ex}}(z)$ for the relaxed case does not display a marked change of sign at large tip-sample distances. Similar differences are also observed in the exchange energy for the relaxed and unrelaxed cases [Fig. 5(c)]. Still, antiferromagnetic alignment $\left(E_{\mathrm{ex}}\right.$ $<0$ ) of the Fe tip with respect to the probed Fe surface atom is energetically much more favorable at small separations. As explained in the previous section, the tip apex atom interacts not only with the probed Fe surface atom but also with the four neighboring $\mathrm{Fe}$ atoms in the surface with antiparallel magnetic moments. Therefore, the negative exchange energy does not exclude ferromagnetic exchange coupling between the magnetic moments of individual Fe atoms.

These differences in the exchange forces and energies are obviously caused by the relaxation of the tip apex atom which depends sensitively on its local magnetic configuration with respect to the approached Fe surface atom [see Fig. 6(a)]. The tip apex atom relaxes toward the surface due to the attractive forces and the shape of the relaxation curve [Fig. 6(a)] resembles the force curves of the apex atom [cf. Fig. 4(a)]. A similar effect is observed for the relaxation of the
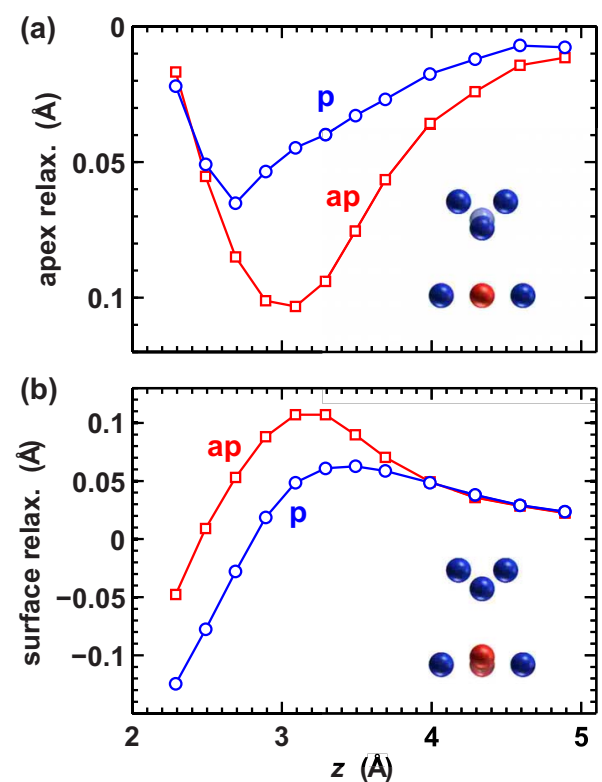

FIG. 6. (Color online) Distance dependence of (a) the vertical tip apex atom relaxation for the five-atom tip and (b) the vertical relaxation of the probed surface Fe atom for the $\mathrm{p}$ and ap alignments, respectively.

surface atom being probed which is attracted toward the tip at large distances and repelled at very close separations [see Fig. 6(b)]. On the ap site, the tip apex atom relaxes about $0.05 \AA$ closer toward the surfaces atom than on the $\mathrm{p}$ site which enhances the exchange interaction that can be inferred from the force curves of Fig. 4(a).

\section{Electronic and magnetic structure changes due to tip-sample interaction}

After analyzing the interaction between tip and sample based on force curves and the resulting relaxations in the previous sections, we now turn to the modifications of the electronic and magnetic structure due to their interaction. One way to monitor the magnetic interaction is to plot the distance dependence of the magnetic moments of tip apex and surface atoms. This is shown in Fig. 7 for the two different magnetic configurations including structural relaxations.

We find that the magnetic moment of the base atoms remains constant at $m_{\text {base }} \approx 3 \mu_{B}$, whereas the apex and surfaceatom moments decrease as the tip approaches the surface. This decrease becomes significant only at separations below $3 \AA$ and is due to an increased hybridization between the states of tip apex and surface atoms. The magnetic-moment drop is more pronounced on the $\mathrm{p}$ site than on the ap site. This result is consistent with the ap configuration (antiferromagnetic coupling) being energetically much more favorable than the p configuration [Fig. 5(c)], as there is a large energy cost to reduce the magnetic moments from their equilibrium values (obtained at large tip-sample separations).

The origin of the magnetic exchange interaction can be traced to the different electronic interactions in the ap and $p$ configurations. In order to study the nature of these interac- 

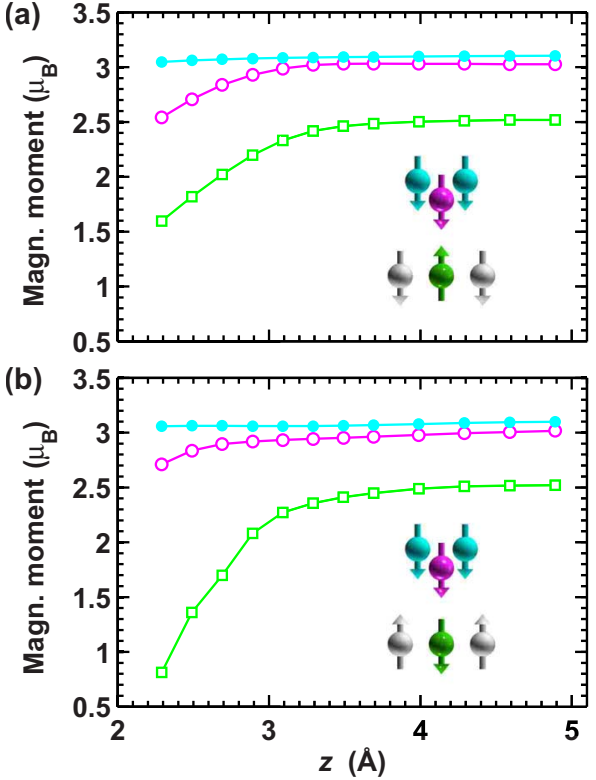

FIG. 7. (Color online) Distance dependence of the absolute magnetic moments of the apex (open circles), base (close circles), and surface (open squares) $\mathrm{Fe}$ atoms in the case of the five-atom $\mathrm{Fe}$ tip for (a) ap and (b) p alignments between the magnetization of tip and surface atoms as shown in the insets including structural relaxations of tip and sample.

tions it is helpful to analyze charge-density difference (CDD) plots for the two types of coupling. This quantity is obtained by subtracting from the charge density of the interacting system consisting of Fe cluster tip and $1 \mathrm{ML} \mathrm{Fe} / \mathrm{W}(001)$ both the charge density of the isolated Fe ML/W(001) and that of the isolated Fe cluster tip, using the relaxed atom positions also for the isolated systems. The CDD plots allow the visualization of the charge transfer associated with the electronic interaction between tip and sample, i.e., the accumulation or depletion of charge.

Figure 8 shows the CDD plots for the ap and p alignments at tip-sample distances of $z=4.9$ and $2.9 \AA$. At large separation, there is a small net charge accumulation between the tip apex atom and the surface Fe atom. Already at this height the interaction depends on the type of spin alignment. The charge accumulation due to tip-sample interaction in the ap configuration is bound to the Fe surface atom and has a node with the Fe apex tip atom. While in the p configuration, it has nodes on both the Fe surface and the tip apex atom. At a very close distance of $z=2.9 \AA$ electronic charge strongly accumulates between the tip apex atom and the surface Fe atoms, implying a strong electronic interaction between the tip and the surface. The charge accumulation in the ap coupling is larger than in the $\mathrm{p}$ coupling in agreement with the ap alignment being energetically more favorable [cf. Fig. 5(c)].

The CDD plots also show that the charge density of the nearest-neighbor $\mathrm{Fe}$ atoms (with respect to the probed $\mathrm{Fe}$ surface atom) is considerably redistributed upon approaching the tip. Therefore, the exchange coupling of these nearestneighbor Fe atoms with the apex atom of the tip plays an important role in determining whether $\mathrm{p}$ or ap alignment is more favorable. Similarly, the redistribution of the base at- om's charge density indicates a significant contribution to the exchange interaction between tip and sample as has been discussed in terms of the exerted exchange forces in Sec. III A [cf. Fig. 4].

\section{Influence of tip size}

One of the more delicate aspects in modeling atomic force microscopy experiments is the geometry used for the tip. Ideally, the tip should consist of thousands of atoms to mimic the tips used in real experiments. However, in practice one is limited by the computational resources required for the calculation. Fortunately, the chemical and magnetic interactions between tip and sample are dominated by the foremost atoms due to the exponential decay of the wave functions while long-range forces can be added based on continuum models. ${ }^{41}$ However, the electronic and magnetic properties at the tip apex is still influenced by the base of the tip used in the model and needs to be investigated.

In the past, theoretical calculations have often been carried out using a single $\mathrm{Fe}$ atom as an idealized model of the tip to study the magnetic exchange force, e.g., on the $\mathrm{NiO}(001)$ surface. ${ }^{31}$ Here, we assess the validity of such a model using the Fe monolayer on W(001) as a test sample by comparing calculations with a single $\mathrm{Fe}$ atom with the fiveatom Fe pyramid tip discussed in the previous sections and an even larger fourteen-atom Fe tip [cf. Fig. 1].

Figure 9(a) displays the calculated force-distance curves for the interaction between the fourteen-atom Fe tip and the Fe ML on W(001). Because of the large tip size and the resulting prohibitive computational effort, we neglected structural relaxations due to the tip-sample interaction in this case. Similar to the calculation with the five-atom Fe tip [cf. Fig. 3], we observed a splitting between the total forces acting on the tip for the $\mathrm{p}$ and ap sites which increases with decreasing tip-sample separation as shown in Fig. 9(a). The resulting exchange force and energy displayed in Fig. 9(b) are negative which indicates a preferred antiferromagnetic coupling with the probed surface Fe atom. This exchange interaction is in good qualitative agreement with the results we obtained for the smaller five-atom Fe tip [cf. Fig. 3].

However, the decomposition of the forces on the distinct tip atoms [Figs. 9(c) and 9(d)] displays interesting discrepancies between the two tip models. The forces acting only on the tip apex atom are qualitatively quite similar, e.g., the forces are larger for ap configuration; however, the splitting between the two curves is larger for the five-atom tip. For the base atoms, there is a more dramatic difference. Here, we obtain a smaller exchange force for the base atoms in the larger tip and the sign of the exchange force on the base atoms is the same as for the apex atom. However, the contribution of all base atoms to the total exchange force is still significant, as seen from $F_{\mathrm{ex}}^{\mathrm{apex}}(z)$ given in Fig. 9(b). For the smaller tip, the sign of the exchange force on the base atoms was opposite to that of the apex atom, thereby reducing the total exchange force. For the fourteen-atom tip their sign is the same, and consequently, the regime of considerable exchange forces sets in at larger tip-sample separations as seen in Fig. 10. 

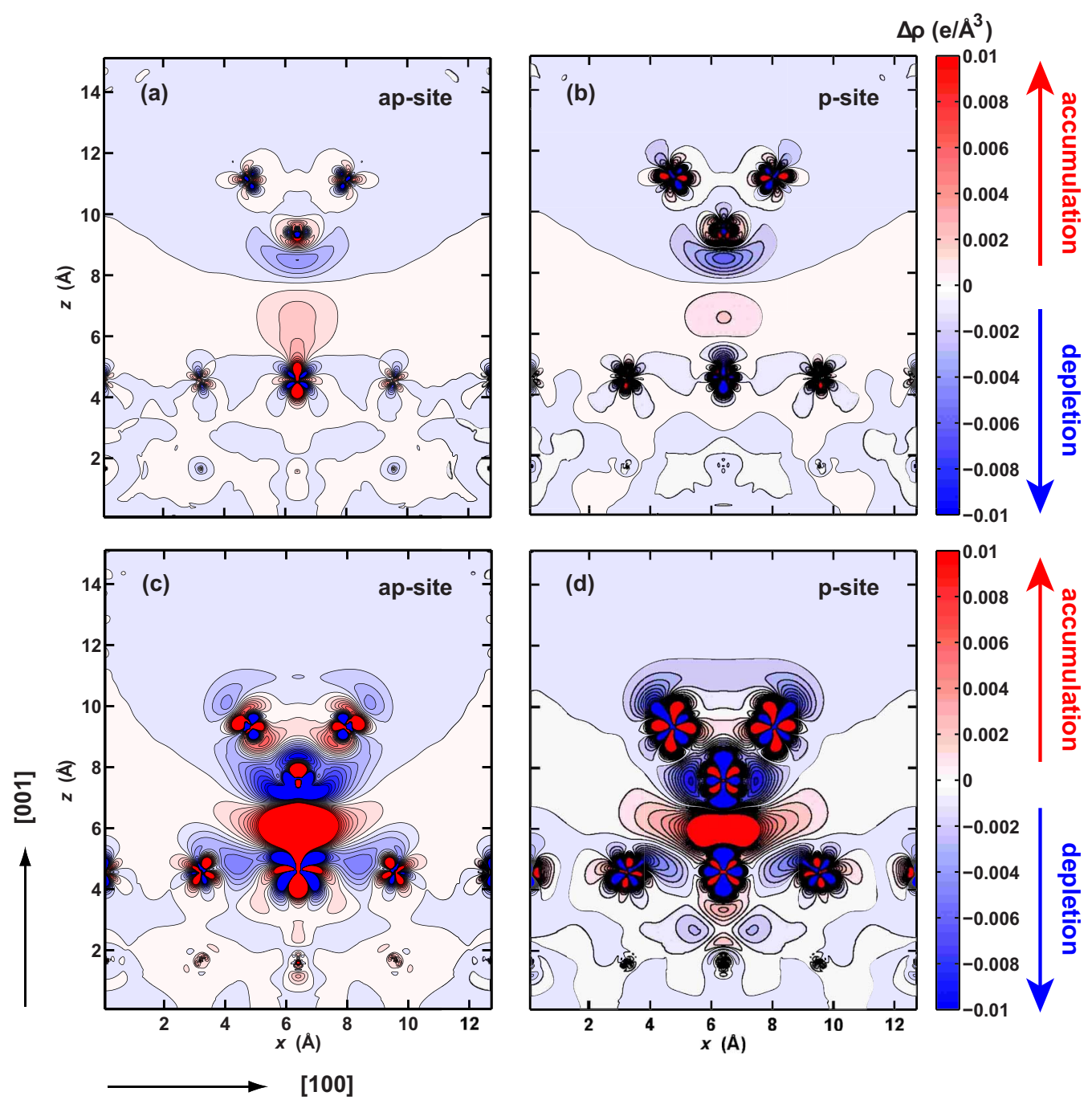

FIG. 8. (Color online) Cross-sectional charge-density difference plots along the [011] direction for the interaction of the five-atom Fe tip (top of each panel) with the Fe monolayer on W(001) (bottom of each panel) at tip-sample separations of $z=4.9 \AA$ for (a) the ap and (b) the $\mathrm{p}$ alignments, and at $z=2.9 \AA$ for (c) the ap and (d) the p alignments. Zones in pale gray (red) and dark gray (blue) denote charge accumulation and depletion, respectively. The results presented here correspond to geometries including relaxations due to tip-sample interaction.

A direct comparison of the exchange forces for different tip models is given in Fig. 10. Obviously, the exchange force obtained for a single $\mathrm{Fe}$ atom tip is even qualitatively different from both pyramid-type tips. At large separations, the exchange forces are much larger than for the pyramid tips while they have the opposite sign at close distance. From these calculations, it is quite clear that a single $\mathrm{Fe}$ atom cannot mimic the exchange forces between a magnetic tip and sample. If we compare the two pyramid-type Fe tips, on the other hand, the general shape of the curve is very similar and the smaller tip gives qualitatively the same result. However, the exchange forces for the bigger Fe tip are significantly enhanced and set in at much larger tip-sample distances which is of crucial importance in experiments.

The dependence of the tip's magnetic moments on the tip-sample separation, displayed in Fig. 11 for the fourteenatom Fe tip, provides additional insight into the tip size dependent magnetic interaction. Similar to the smaller fiveatom tip, only the magnetic moment of the foremost tip atom is reduced upon the approach to the surface. However, the moment of the apex atom is smaller [cf. Fig. 1], and its relative reduction due to tip-sample interaction is slightly enhanced for the larger tip. In contrast, the magnetic moment of a single $\mathrm{Fe}$ atom tip is practically constant upon approaching the surface (not shown) and the exchange force is smaller [cf. Fig. 10]. Therefore, we conclude that a large magnetic moment of the apex atom does not guarantee significant magnetic exchange forces. Instead, a less rigid magnetic moment of the apex atom, i.e., tunable in size due to the interaction, is favorable in detecting large exchange forces. In addition, the exchange forces on the base atoms of the tip play an important role for the total exchange force as discussed above.

In order to check the influence of using the larger fourteen-atom pyramid tip on the relaxations, we performed a structural optimization for a single tip-sample distance of $z=2.9 \AA$ A. As for the five-atom tip, we find that due to the attractive interaction the apex atom relaxes toward the sur- 

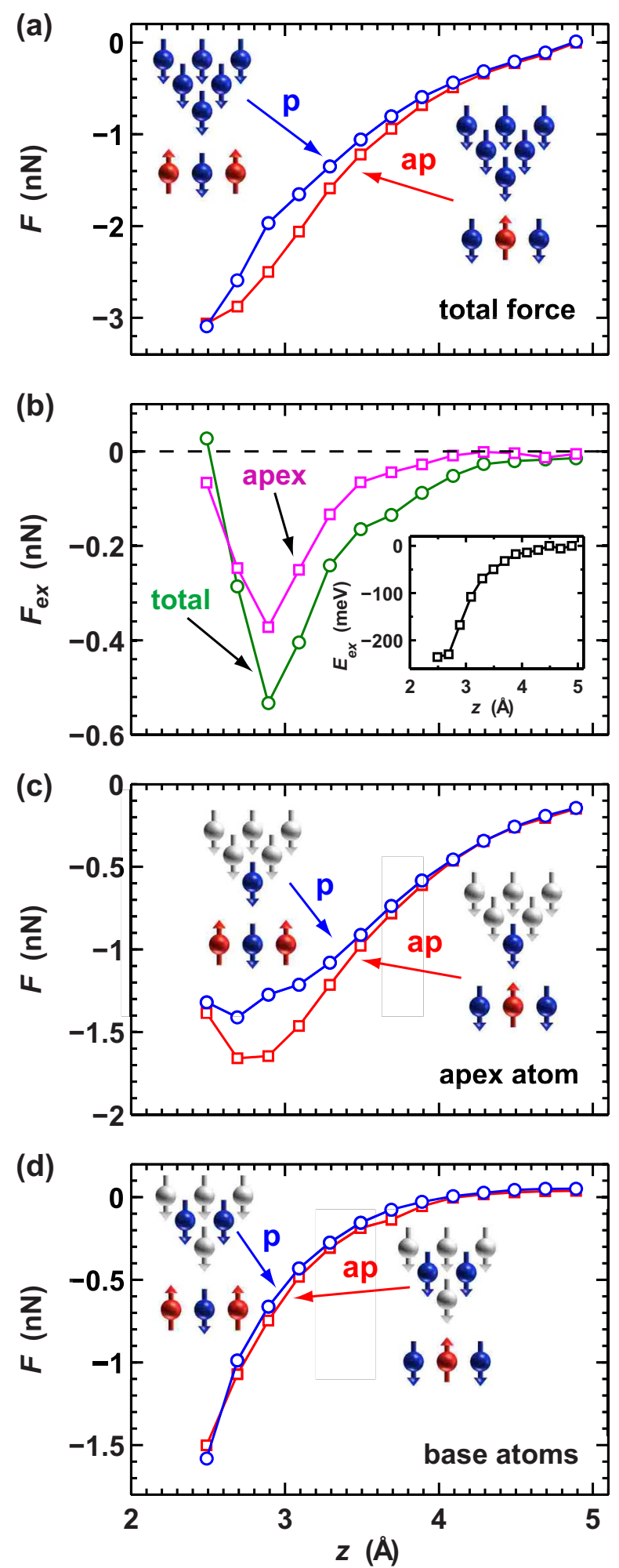

FIG. 9. (Color online) (a) Calculated force curves between an $\mathrm{Fe}$ tip consisting of fourteen atoms (cf. Fig. 1), and an Fe ML on $\mathrm{W}(001)$ for parallel (p) and antiparallel (ap) alignments of the tip magnetization and the probed Fe surface atom. Structural relaxations due to tip-sample interaction have been neglected in this case. (b) Total exchange force on the tip and exchange force on the apex atom. The inset shows the exchange energy. (c) Forces acting on the apex atom of the tip. (d) Forces acting on the base atoms of the tip.

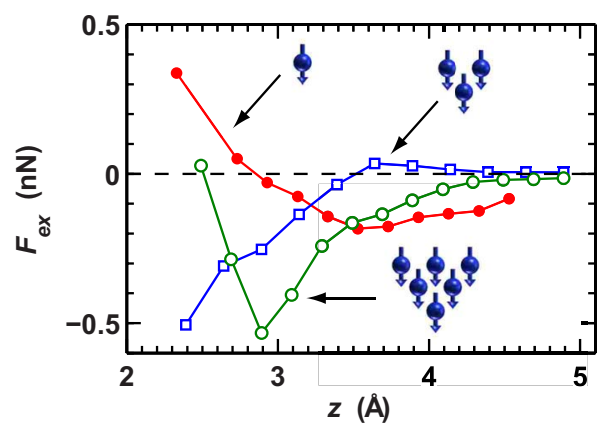

FIG. 10. (Color online) Comparison of the magnetic exchange force $F_{\text {ex }}(z)$ as a function of tip-sample separation $z$ on the $\mathrm{Fe}$ monolayer on W(001) using a single-atom Fe tip, a five-atom Fe tip, and an Fe tip consisting of fourteen atoms, as shown in Fig. 1. All force curves presented in this plot have been obtained without structural relaxations of tip and sample due to their interaction.

face. The relaxation values for the five-atom $\mathrm{Fe}$ cluster tip are 0.11 and $0.05 \AA$ for the ap and p alignments, respectively, while for the fourteen-atom tips the values are 0.16 and $0.12 \AA$ for the ap and p alignments, respectively. The difference in the relaxations are in nice qualitative agreement for the five- and fourteen-atom tips, in particular, the apex atom relaxes more in the ap than in the $\mathrm{p}$ alignment. At this close tip-sample separation of $2.9 \AA$, the total exchange force on the fourteen-atom tip after relaxation is nearly unchanged while it actually decreases for the five-atom tip [cf. Fig. 5(b)].

Further evidence for the modified tip-sample interaction of a single-atom tip and multiatom tips can be obtained by
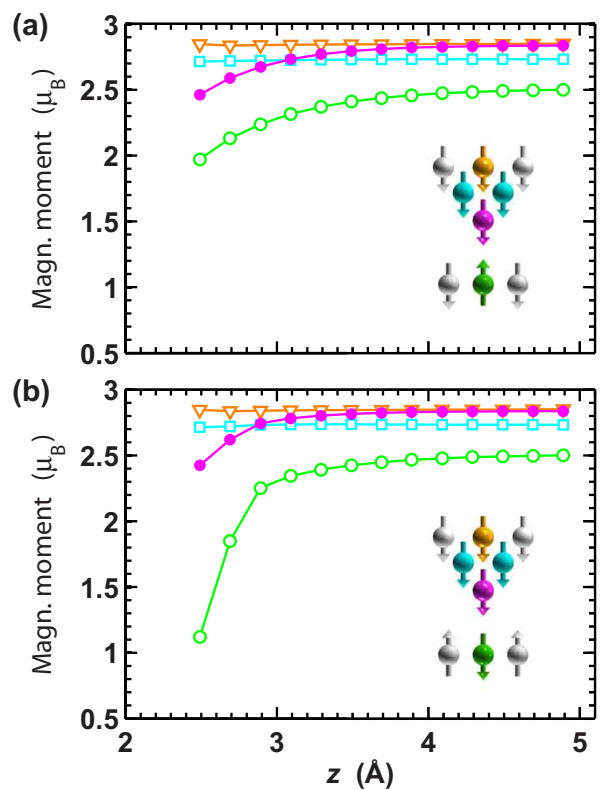

FIG. 11. (Color online) Distance dependence of the magnetic moments of different $\mathrm{Fe}$ atoms of the fourteen-atom tip-apex (closed circles), base second layer (open squares), and center atom of third layer (open triangles) - and the surface $\mathrm{Fe}$ atom (open circles) of the Fe ML on W(001) for (a) ap and (b) p alignments between the magnetization of tip and surface atom as shown in the insets without structural relaxations of tip and sample. 

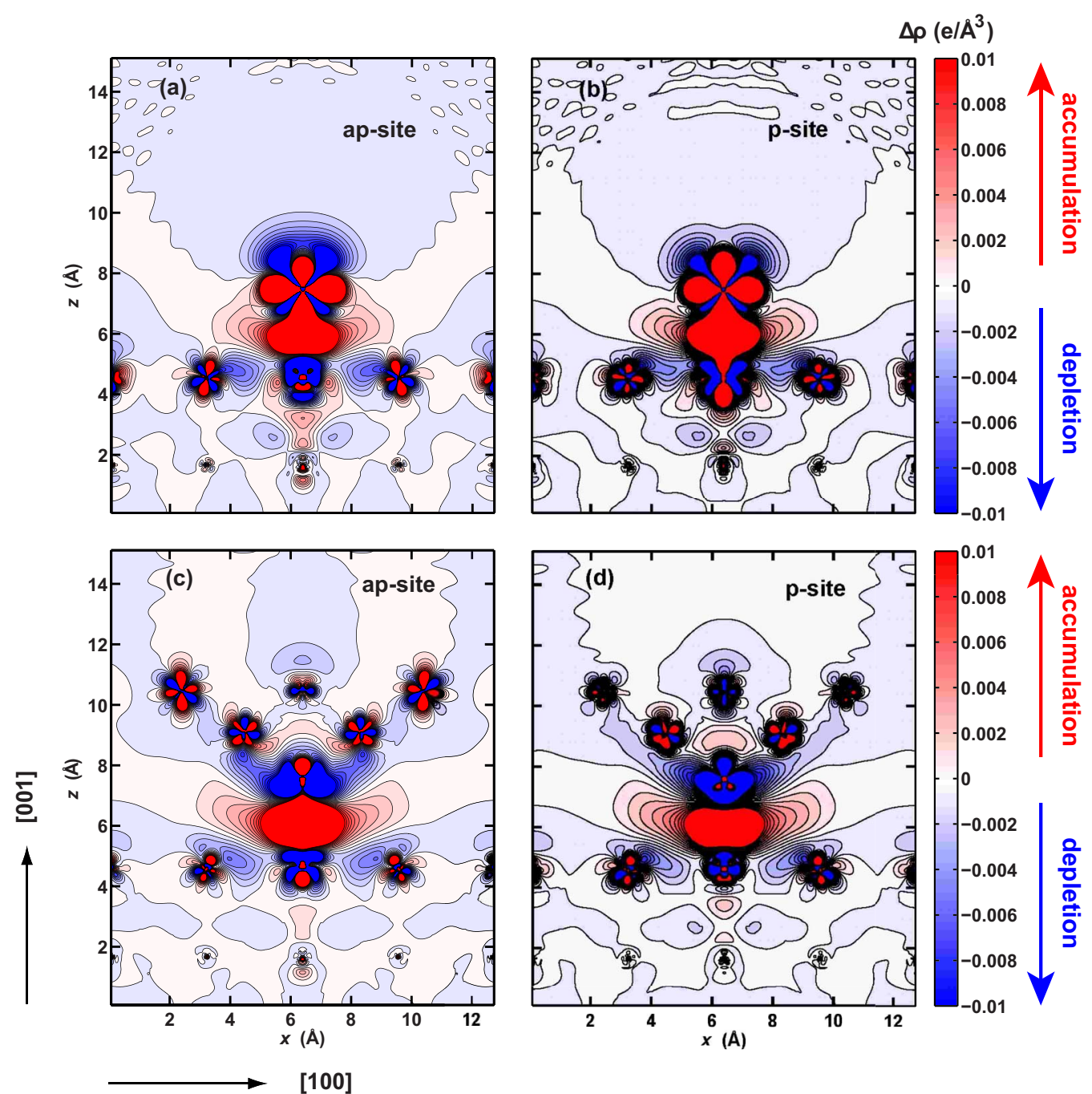

FIG. 12. (Color online) Cross-sectional charge-density difference plots along the [011] direction for the interaction of the single Fe atom tip (top of each panel) with the Fe monolayer on W(001) (bottom of each panel) at a tip-sample separation of $z=2.9 \AA$ for (a) the ap coupling and (b) the p coupling. (c) and (d) show equivalent plots for the Fe tip consisting of fourteen atoms. Zones in pale gray (red) and dark gray (blue) denote charge accumulation and depletion, respectively. The results presented here correspond to unrelaxed geometries for the single Fe atom tip and relaxed geometries for the fourteen-atom Fe tip.

examining the CDD plots. These are shown in Fig. 12 for the single-atom $\mathrm{Fe}$ and fourteen-atom $\mathrm{Fe}$ tips at a separation of $z=2.9 \AA$ which can be directly compared to those for the five-atom tip, displayed in Figs. 8(c) and 8(d). These graphs show a similar redistribution of electronic charge density between tip apex and surface atoms for the two multiatom tips upon approaching the tip to the surface. The dependence on the two types of spin alignment is also quite similar. Interestingly, the charge redistribution of the base atoms does not depend as dramatically on the type of coupling for the fourteen-atom tip as for the five-atom tip. This can explain the smaller exchange forces acting on the base atoms for the larger tip. In contrast, the electronic charge distribution for the single-atom tip, shown in Figs. 12(a) and 12(b), is distinctively different from the case of the pyramid-type tips. In fact, the CDD plots for the ap and p alignments of the singleatom tip are very similar, which explains the very small exchange force at $z=2.9 \AA$ for the single-atom tip observed in Fig. 10. Therefore, one should use multiatom tips in ab initio simulations of magnetic exchange force microscopy in order to properly describe the electronic and magnetic properties of the tip as well as the magnetic interaction with the sample.

\section{E. Implications for spin-polarized STM}

So far, we have interpreted our calculations on the magnetic tip-sample interaction only with respect to magnetic exchange force microscopy. However, a similar situation occurs in a spin-polarized STM experiment which relies on measuring the spin-polarized tunneling current between a magnetic tip and a magnetic sample.

In the constant-current mode the current is fixed while scanning the tip across the surface by approaching or retracting the tip in the vertical direction. Due to the variation in the spin-polarized local density of states in the vacuum, the constant-current mode allows resolving of magnetic structures on the atomic scale, ${ }^{3,11}$ e.g., the antiferromagnetic order of the Fe monolayer on W(001). ${ }^{6}$ In simulations of STM 
experiments, one often neglects structural relaxations of the tip while it is scanned across the sample. However, in some cases they can lead to large enhancements of the corrugation amplitude, ${ }^{12-14}$ i.e., the maximum vertical variation in tip position while it is scanned across the surface.

As we have seen in Sec. III B, tip relaxations can depend on the local magnetic configuration between tip and sample magnetizations. As the tunneling current depends exponentially on the tip-sample separation and is dominated by the foremost tip atom, a relaxation of the apex atom can drastically change the measurable corrugation amplitude. In a simple model, we can assume that the tunneling current depends exponentially on the actual distance between the tip apex atom and the surface atom underneath it which we denote by $z_{\mathrm{a}-\mathrm{s}}$. This distance is given by

$$
z_{\mathrm{a}-\mathrm{s}}(z)=z-\Delta z_{\mathrm{a}}(z)-\Delta z_{\mathrm{s}}(z),
$$

where $z$ is the nominal separation without relaxations, and $\Delta z_{\mathrm{a}}(z)$ and $\Delta z_{\mathrm{s}}(z)$ denote the relaxations of tip apex and surface atom, respectively. These relaxations are displayed in Fig. 6 for the $\mathrm{p}$ and ap alignments of the magnetic moments of the apex atom of the five-atom Fe tip and the probed $\mathrm{Fe}$ surface atom of the Fe ML on W(001). The actual separation between the apex and the surface atom is plotted in Fig. 13(a) as a function of the nominal distance $z$ for the ap and $p$ configurations. Quite obviously, there is a significant deviation from the linear unrelaxed case, which indicates that the relaxation are significant. Also a clear difference between the curves for the $\mathrm{p}$ and ap sites can be observed.

In order to obtain a constant tunneling current, $z_{\mathrm{a}-\mathrm{s}}$ must be the same on both the $p$ and ap sites of the surface. Therefore, the corrugation amplitude $\Delta z=z_{\mathrm{ap}}-z_{\mathrm{p}}$ due to structural relaxations is given by

$$
\Delta z(z)=\left[\Delta z_{\mathrm{a}}^{\mathrm{ap}}(z)+\Delta z_{\mathrm{s}}^{\mathrm{ap}}(z)\right]-\left[\Delta z_{\mathrm{a}}^{\mathrm{p}}(z)+\Delta z_{\mathrm{s}}^{\mathrm{p}}(z)\right],
$$

where the upper index denotes the relaxations on the ap and p sites, respectively. This quantity is shown in Fig. 13(b) as a function of the unrelaxed apex-surface distance $z$ between the Fe tip and the Fe ML on W(001). There is a very steep rise of this apparent corrugation amplitude below a nominal tip-sample distance of $4 \AA$ at which it is 2 pm while beyond this distance, the effect of structural relaxations becomes very small. The SP-STM experiments on the Fe monolayer on W(001) (Refs. 6 and 42) reported corrugation amplitudes between 3 and $10 \mathrm{pm}$. The absolute tip-sample distance is unknown in STM; however, in some cases the tunneling parameters, i.e., bias voltage and tunneling current, hint at small separations. Therefore, our results indicate that contributions due to structural relaxations of the tip may play a role in some SP-STM measurements.

In our discussion, we have so far neglected that the spinpolarized tunneling current is different on the two magnetic surface sites of the Fe ML on W(001). This contribution to the current depends on the spin polarization of the local density of states of tip and sample close to the Fermi energy. In the constant-current mode it causes a different tip-sample separation on the two Fe surface atoms of opposite spin direction and allows the resolution of the atomic-scale magnetic structure. ${ }^{6}$ For the total corrugation amplitude both ef-
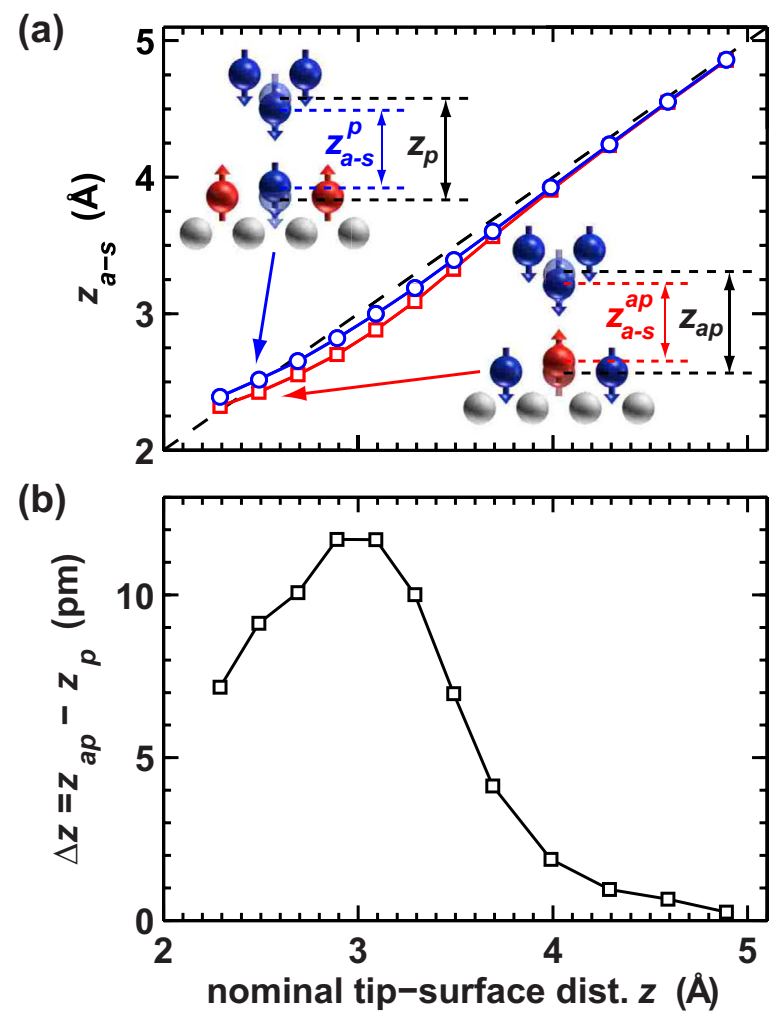

FIG. 13. (Color online) (a) Actual separation between the tip apex atom of the five-atom Fe tip and the surface atom of the Fe monolayer on $\mathrm{W}(001)$ including structural relaxations, $z_{\mathrm{a}-\mathrm{s}}$, as a function of the separation before relaxation, $z$ (cf. inset) for the $\mathrm{p}$ and ap alignments between the magnetic moments of tip apex and surface atom. Dashed line indicates the unrelaxed case. (b) Effective corrugation amplitude which would occur in a spin-polarized STM experiment due to the magnetic configuration dependent tip and sample relaxations.

fects, i.e., spin polarization of the tunneling current and spinalignment dependent relaxations, are additive. However, the two contributions can be of opposite sign as the first depends on the spin polarization at the Fermi energy while the latter stems from all occupied states. Therefore, the corrugation amplitude due to structural relaxations can either enhance or diminish the corrugation from the spin-polarized current.

\section{SUMMARY}

We have performed first-principles calculations based on density-functional theory to study the interaction between a magnetic tip and a magnetic sample which occurs in magnetic exchange force microscopy (MExFM). We have studied tips consisting of one to fourteen $\mathrm{Fe}$ atoms and have chosen one monolayer Fe on $\mathrm{W}(001)$ as a sample system which exhibits an antiferromagnetic checkerboard structure and has been resolved on the atomic scale by both MExFM (Ref. 38) and spin-polarized scanning tunneling microscopy (SP-STM) (Ref. 6).

Our calculations clearly demonstrate the inadequacy of using a single magnetic atom as a model for the magnetic tip in MExFM as the obtained force curves differ even qualita- 
tively from those of cluster tips. Increasing the size of our Fe bcc(001)-type pyramid tip still leads to quantitative changes; however, qualitatively the five- and fourteen-atom Fe tips exhibit the same features in the force-distance curves and lead to antiferromagnetic exchange coupling with the probed Fe surface atom being energetically more stable. Quantitatively, we observe that the onset of significant exchange forces is shifted to larger tip-sample separations for the larger tip.

The exchange forces on the apex atom is the dominant contribution to the total exchange force for both tips but contributions from other tip atoms cannot be neglected and may even reduce the total exchange force. This effect is especially pronounced for small tips. The chemical and magnetic interactions of the $\mathrm{Fe}$ apex atom are significant with both the Fe surface atom underneath it as well as the nearestneighbor Fe surface atoms.

We find that structural relaxations of tip and sample due to their chemical and magnetic interactions play an important role and can greatly enhance the measurable MExFM signal.
These relaxations depend on the local magnetic configuration of tip and sample magnetizations, and can have an influence on SP-STM experiments as well. In particular, the effective corrugation amplitude of the magnetic superstructure observable in SP-STM can be enhanced or diminished.

\section{ACKNOWLEDGMENTS}

It is our pleasure to thank S. Blügel, Y. Mokrousov, P. Ferriani, A. Schwarz, U. Kaiser, R. Schmidt, and R. Wiesendanger for many insightful discussions. Computations were performed at the Hamburg University of Technology, the Norddeutscher Verbund für Hoch-und Höchstleistungsrechnen (HLRN), and the Forschungszentrum Jülich (JUMP). We acknowledge financial support from the DFG (Grants No. HO 2237/3-1 and No. HE 3292/4-1). S.H. thanks the Stifterverband für die Deutsche Wissenschaft and the Interdisciplinary Nanoscience Center Hamburg for financial support.
*Corresponding author; clazo@physnet.uni-hamburg.de

${ }^{1}$ I. S. Osborne, Science 294, 1483 (2001).

${ }^{2}$ M. R. Freeman and B. C. Choi, Science 294, 1484 (2001).

${ }^{3}$ S. Heinze, M. Bode, A. Kubetzka, O. Pietzsch, X. Nie, S. Blügel, and R. Wiesendanger, Science 288, 1805 (2000).

${ }^{4}$ H. Yang, A. R. Smith, M. Prikhodko, and W. R. L. Lambrecht, Phys. Rev. Lett. 89, 226101 (2002).

${ }^{5}$ M. Bode, Rep. Prog. Phys. 66, 523 (2003).

${ }^{6}$ A. Kubetzka, P. Ferriani, M. Bode, S. Heinze, G. Bihlmayer, K. von Bergmann, O. Pietzsch, S. Blügel, and R. Wiesendanger, Phys. Rev. Lett. 94, 087204 (2005).

${ }^{7}$ U. Kaiser, A. Schwarz, and R. Wiesendanger, Nature (London) 446, 522 (2007).

${ }^{8}$ R. Wiesendanger, D. Bürgler, G. Tarrach, A. Wadas, D. Brodbeck, G. G. H.-J. Güntherodt, R. J. Gambino, and R. Ruf, J. Vac. Sci. Technol. B 9, 519 (1991).

${ }^{9}$ Note that MExFM is not the same as magnetic force microscopy (Ref. 43), which is well suited to exploring ferromagnetic domain structures but cannot achieve atomic resolution because data acquisition involves the sensing of long-range magnetostatic forces between tip and sample. By using an atomic force microscope with a magnetic tip, MExFM makes it possible to detect the short-range magnetic exchange force between tip and sample spins. Thus it has resolution at the atomic spin level.

${ }^{10}$ J. Tersoff and D. R. Hamann, Phys. Rev. Lett. 50, 1998 (1983).

${ }^{11}$ D. Wortmann, S. Heinze, P. Kurz, G. Bihlmayer, and S. Blügel, Phys. Rev. Lett. 86, 4132 (2001).

${ }^{12}$ M. Di Ventra and S. T. Pantelides, Phys. Rev. B 59, R5320 (1999).

${ }^{13}$ W. A. Hofer, A. J. Fisher, R. A. Wolkow, and P. Grütter, Phys. Rev. Lett. 87, 236104 (2001).

${ }^{14}$ J. M. Blanco, C. González, P. Jelínek, J. Ortega, F. Flores, and R. Pérez, Phys. Rev. B 70, 085405 (2004).

${ }^{15}$ R. Pérez, M. C. Payne, I. Štich, and K. Terakura, Phys. Rev. Lett. 78, 678 (1997).
${ }^{16}$ R. Pérez, I. Stich, M. C. Payne, and K. Terakura, Phys. Rev. B 58, 10835 (1998).

${ }^{17}$ N. Sasaki, S. Watanabe, and M. Tsukada, Phys. Rev. Lett. 88, 046106 (2002).

${ }^{18}$ A. S. Foster, A. L. Shluger, and R. M. Nieminen, Appl. Surf. Sci. 188, 306 (2002).

${ }^{19}$ F. J. Giessibl, Rev. Mod. Phys. 75, 949 (2003).

${ }^{20}$ A. S. Foster, A. L. Shluger, and R. M. Nieminen, Nanotechnology 15, S60 (2004).

${ }^{21}$ M. B. Watkins and A. L. Shluger, Phys. Rev. B 73, 245435 (2006).

${ }^{22}$ V. Caciuc, H. Hölscher, S. Blügel, and H. Fuchs, Phys. Rev. Lett. 96, 016101 (2006).

${ }^{23}$ Y. Sugimoto, P. Pou, M. Abe, P. Jelinek, R. Pérez, S. Morita, and O. Custance, Nature (London) 446, 64 (2007).

${ }^{24}$ N. Atodiresei, V. Caciuc, S. Blügel, and H. Hölscher, Phys. Rev. B 77, 153408 (2008).

${ }^{25}$ V. Caciuc, H. Hölscher, D. Weiner, H. Fuchs, and A. Schirmeisen, Phys. Rev. B 77, 045411 (2008).

${ }^{26}$ H. Ness and F. Gautier, Phys. Rev. B 52, 7352 (1995).

${ }^{27}$ K. Nakamura, H. Hasegawa, T. Oguchi, K. Sueoka, K. Hayakawa, and K. Mukasa, Phys. Rev. B 56, 3218 (1997).

${ }^{28}$ K. Nakamura, T. Oguchi, H. Hasegawa, K. Sueoka, K. Hayakawa, and K. Mukasa, Appl. Surf. Sci. 142, 433 (1999).

${ }^{29}$ A. S. Foster and A. L. Shluger, Surf. Sci. 490, 211 (2001).

${ }^{30}$ H. Momida and T. Oguchi, J. Phys. Soc. Jpn. 72, 588 (2003).

${ }^{31}$ H. Momida and T. Oguchi, Surf. Sci. 590, 42 (2005).

${ }^{32}$ H. Hosoi, K. Sueoka, K. Hayakawa, and K. Mukasa, Appl. Surf. Sci. 157, 218 (2000).

${ }^{33}$ H. Hosoi, K. Sueoka, and K. Mukasa, Nanotechnology 15, 505 (2004).

${ }^{34}$ H. Hölscher, S. M. Langkat, A. Schwarz, and R. Wiesendanger, Appl. Phys. Lett. 81, 4428 (2002).

${ }^{35}$ S. M. Langkat, H. Hölscher, A. Schwarz, and R. Wiesendanger, Surf. Sci. 527, 12 (2003). 
${ }^{36}$ M. Schmid, J. Mannhart, and F. J. Giessibl, Phys. Rev. B 77, 045402 (2008).

${ }^{37}$ U. Kaiser, A. Schwarz, and R. Wiesendanger, Phys. Rev. B 78, 104418 (2008).

${ }^{38}$ R. Schmidt, C. Lazo, H. Hölscher, U. H. Pi, V. Caciuc, A. Schwarz, R. Wiesendanger, and S. Heinze, Nano Lett. (to be published).

${ }^{39}$ J. P. Perdew, K. Burke, and M. Ernzerhof, Phys. Rev. Lett. 77, 3865 (1996)
${ }^{40}$ G. K. H. Madsen, P. Blaha, K. Schwarz, E. Sjöstedt, and L. Nordström, Phys. Rev. B 64, 195134 (2001).

${ }^{41}$ F.-J. Giessibl, Phys. Rev. B 56, 16010 (1997).

${ }^{42}$ M. Bode, E. Y. Vedmedenko, K. von Bergmann, A. Kubetzka, P. Ferriani, S. Heinze, and R. Wiesendanger, Nat. Mater. 5, 477 (2006).

${ }^{43}$ Y. Martin and H. K. Wickramasinghe, Appl. Phys. Lett. 50, 1455 (1987). 